\title{
La institución educativa en la configuración de las expectativas de los alumnos
}

DOI: https://doi.org/10.32870/dse.v0i8.310

\section{Rosa Angélica Martínez Téllez*}

Resumen: La escuela como la institución donde los estudiantes interactúan, socializan y construyen su identidad a través de la experiencia escolar, influye en la elaboración de metas, ideales y anhelos. Es por ello que el siguiente proyecto buscó identificar cuáles son las expectativas de futuro en jóvenes del bachillerato tecnológico CETIS 161 en Tala, Jalisco; se analiza la escuela como institución motivadora para generar proyectos exitosos de vida, teniendo como base la cultura y el contexto actual de los jóvenes. Palabras clave: estudiantes, jóvenes, cultura, educación, bachillerato tecnológico.

\begin{abstract}
The school as an institution where students interact, socialize and build their identity through school experience influences the development of goals, ideals and aspirations. The next project looking for to identify what are the expectations of future technological high school youngs CETIS 161 in Tala, Jalisco, we analyze the school as an institution motivating to generate successful projects of life, based on the culture and context of young people today. Key words: Students, youth, culture, education, technical high school.
\end{abstract}

El artículo analiza a jóvenes, se centra en los que pertenecen a un contexto escolar, debido a que los estudiantes definen su identidad a través de los procesos de socialización, gustos, intereses y modelos a seguir. Los autores Dubet y Martuccelli (1999) plantean que los alumnos utilizan tres lógicas de acción en su relación con la escuela: "la integración en la cual los estudiantes configuran su mundo personal en función de los intereses y el mundo escolar: la subjetivación, donde la adquisición del saber (aceptación de la escuela) se da a la par de la construcción de un futuro personal, de una personalidad y la estrategia, donde se ponen en juego diversas habilidades y saberes que permiten un manejo exitoso del mundo escolar" (citado por Ávalos, 2007: 05). Por lo tanto durante ese proceso de interacción, los estudiantes reflexionarán y construirán sus ideales de futuro, basados en un capital escolar que están adquiriendo.

La institución educativa en su función formativa, reproduce hábitos, valores, creencias que se transmiten de generación en generación. Bourdieu y Passeron (1981: 26) expresan que toda acción pedagógica es objetivamente una violencia simbólica en cuanto impone, a través de un poder arbitrario, una arbitrariedad cultural. Cada sistema de enseñanza institucionalizada, debe las

* Maestra en Comunicación de la Ciencia y Cultura. Profesora e investigadora en el CETIS 161 de Tala, Jalisco y TecMilenio en Guadalajara, Jalisco; correo electrónico: angelicamartinez86@gmail.com 
características específicas de su estructura y de su funcionamiento al hecho de que debe producir y reproducir, a través de los medios propios de la institución; es decir, la escuela no tiene valor por sí misma, su valor depende de la creencia socialmente producida, entre los grupos y las clases.

La cultura influye en nuestras ideas, valores, actos, Clifford Geertz (1966) señala que se comprende mejor no como complejos esquemas concretos de conducta, sino como una serie de mecanismos de control, planes, recetas, fórmulas, reglas instrucciones, que gobiernan la cultura; la humanidad depende de esos mecanismos de control que rigen su conducta. La escuela ha sido la institución legítima de reproducción de los saberes, de homogenización de la cultura. Bordieu y Paseron (1981) señalan que el origen social define las posibilidades de escolarización, determinan modos de vida y de trabajo; es por ello que la institución educativa va a jugar un papel muy importante al momento de educar y preparar a los jóvenes para diseñar, planear e idealizar su futuro; en el bachillerato se encuentran en una etapa de toma de decisiones relacionas con su vida académica, laboral y personal.

La perspectiva de que parte el siguiente proyecto es sociocultural, estudia la institución educativa (estructura), a los estudiantes (sujetos) y la cultura. El ser humano se ha preocupado por el tiempo como uno de los elementos que dan sentido a su vida, recordar el pasado, vivir el presente y planear el futuro. El divulgador Paul Davies (1996: 22) afirmó que mucho de lo que creemos acerca del tiempo es resultado de un condicionamiento cultural. Nosotros nos enfocaremos en el tiempo por venir, en el futuro, específicamente en las expectativas, las cuales el psicólogo Bandura (1999) las definió como la probabilidad de alcanzar una meta concreta. La relación que existe entre la institución educativa y la configuración de expectativas de futuro, se encuentra en que lo que señala Toffler (1973: 422), en la escuela se producen imágenes sucesivas y alternativas del futuro, presunciones sobre las clases de trabajo, profesiones y vocaciones que necesitaremos dentro de veinte o cincuenta años. Los modelos culturales que asocian determinadas profesiones y opciones escolares a un determinado medio social. Otro autor (Dator, 2005: 2) nos dice que los estudios de futuro se concentran en "las imágenes del futuro" que cada individuo y grupo tienen. Es decir, las expectativas de futuro se producen en la mente de cada uno de los individuos, pero se legitiman a partir de la colectividad. La principal relación que trató de develar esta investigación fue la que se teje entre la educación y las expectativas de futuro.

Las expectativas de futuro se van a materializar a través de diferentes discursos, los cuales tienen la finalidad de producir y fijar legitimidades, validaciones y publicidades (Angenot, 2010); los discursos legítimos contribuyen a normalizar prácticas y maneras de ver; es decir, nos indican cuáles serán las profesiones socialmente aceptadas, así como los estilos de vida deseables. En esas maneras de ver se hacen presentes diferentes redes sociales a las que pertenecen los jóvenes como la familia, la escuela, la política, entre otras.

La institución se seleccionó debido a que es un bachillerato tecnológico; es decir, ofertan diferentes carreras técnicas, diseñadas para insertar a sus egresados en el mundo laboral, aunque 
también pueden continuar estudiando si así lo desean, los egresados adquieren certificado y cédula técnica. Guerra (2000) realizó una comparación entre los alumnos del bachillerato tecnológico y el resto de los alumnos que estudian otro bachillerato, entre los resultados encontró que los jóvenes del bachillerato tecnológico enfrentan condiciones más adversas tanto para mantener la situación de escolarizados como para continuar estudiando, desventajas económicas, experiencias de rechazo en otras escuelas y necesidades de trabajo para sostener sus estudios. La investigación se centró en estudiantes del bachillerato tecnológico CETIS 161, para conocer cuáles son las expectativas de futuro.

El bachillerato tecnológico CETIS 161 se encuentra ubicado en el municipio de Tala, Jalisco, en la calle Juárez N. 10. Col. 20 de noviembre. La muestra se formó con un total de 100 cuestionarios aplicados a los alumnos de las carreras técnicas que ofrece la institución. El cuestionario se contestó de forma presencial el día 21 de marzo, también la encuesta estuvo en línea desde el día 20 de marzo al $1^{\circ}$ de abril de 2013.

A continuación se presentan los resultados que se consideraron más importantes para tejer la relación entre los estudiantes, la institución educativa y las expectativas de futuro. El mayor porcentaje de alumnos que contestaron las encuestas se concentraron en la especialidad de Programación, en la segunda posición se colocaron los laboratoristas químicos, posteriormente apareció Mantenimiento Automotriz y en menor cantidad Contabilidad.

Las estadísticas mostraron que las mujeres tuvieron una mayor participación al obtener 61 por ciento, mientas que los hombres alcanzaron 39 por ciento. De acuerdo a la especialidad y al género, se pudo observar que en la mayoría de las especialidades las mujeres tuvieron una mayor participación, a excepción de la carrera de Mantenimiento Automotriz, donde todos los sujetos encuestados fueron hombres.

La mayoría de los alumnos, oscilan entre los 17 y 18 años; sin embargo, también se encontraron estudiantes de 19 y 20 años, en menor cantidad. Se identificó que más de la mitad de la población estudiantil se encuentra en el municipio de Tala, mientras que el 48 por ciento pertenecen a 12 municipios cercanos, estos alumnos viajan diariamente para tener acceso a la educación en el CETIS, en mayor número se concentra en el Refugio con un 13 por ciento, en Ruiseñores el 8 por ciento y en tercer lugar se encontró un empate con tres municipios: Ahuisculco, La Venta y Navajas.

Los estudios situados en contextos de pobreza, drogadicción y situaciones de riesgo han demostrado que los jóvenes no se preocupan o visualizan un futuro, porque se encuentran enfocados en el presente, en el aquí y el ahora (Garro Rojas, 2006); De la O y Flores Ávila, 2011); sin embargo, en contextos educativos, se encontró que 93 por ciento de los encuestados sí piensa constantemente en su futuro, mientras que sólo, 6 por ciento contestó que no y el uno por ciento no contestó. Los estudiantes encuestados colocan el futuro como un tema de reflexión, planeación y decisión. Los alumnos de sexto semestre se encuentran próximos a egresar, es un momento de su vida donde deben tomar decisiones importantes como continuar estudiando, ingresar al ámbito laboral o combinar ambos. 
Los estudiantes fueron cuestionados sobre los elementos con los que relacionan el futuro, a continuación se muestra un comparativo entre hombres y mujeres, el cual muestra que en el primer lugar hay coincidencias, consideran que un elemento importante en el futuro es tener un buen trabajo, cabe mencionar que los hombres evaluaron con el mismo porcentaje tener éxito, la segunda posición para los hombres fue formar una familia, estudiar y trabajar y tener estabilidad económica, mientras que para las mujeres fue tener estabilidad económica.

Gráfica 1. Comparativo de las dimensiones sobre el futuro

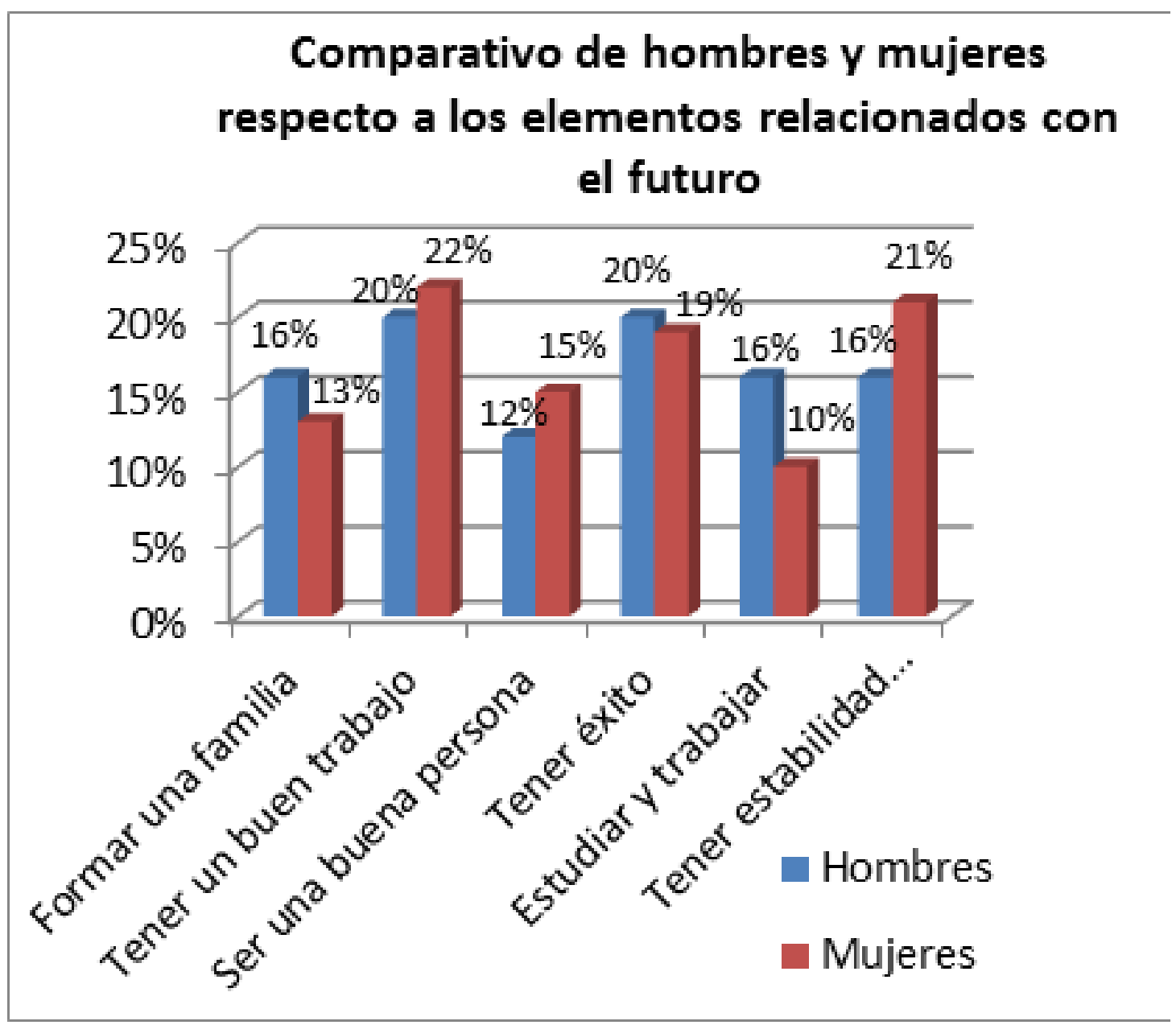

Fuente: elaboración propia, con los datos obtenidos en la encuesta. 
Los estudiantes también fueron cuestionados sobre las diferentes temáticas a las cuales les dedican mayor reflexión en torno al futuro, los alumnos coinciden en que el trabajo es la principal dimen sión en la cual reflexionan; el segundo lugar varía de acuerdo al género, en el caso de las mujeres piensan bastante en la escuela, mientras los hombres optan por formar su propia familia; la tercera posición también muestra variaciones, las mujeres se interesan por la creación de su propia familia, mientras que los hombres se concentran en la diversión; en el cuarto lugar, en el caso de las mujeres, se posicionó la diversión, mientras que para los hombres se colocó la escuela, en último lugar los jóvenes colocaron la dimensión del noviazgo.

Gráfica 2 Comparativo dimensiones del futuro

\section{Comparativo dimensiones de futuro de mayor importancia}

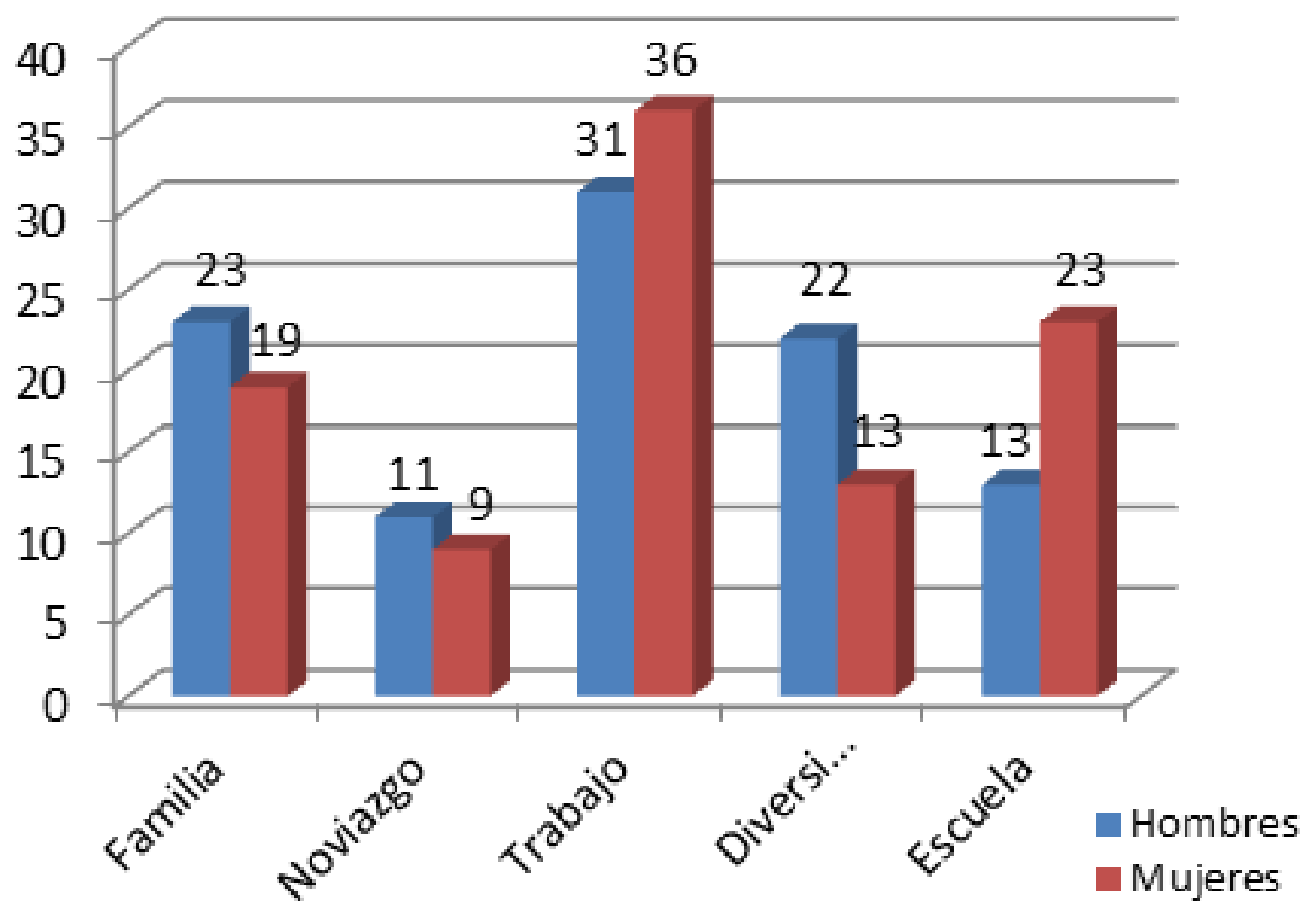

Fuente: elaboración propia, con los datos obtenidos en la encuesta. 


\section{Estudiantes y futuro}

Las respuestas enfocadas en la definición del futuro mostraron diferentes aristas, en el caso de los hombres encuestados se encontró que visualizan el futuro como algo positivo, que va a mejorar su calidad de vida, algo estable, chido, bueno; también sobresale un aspecto negativo o pesimista considerando al futuro inútil, trabajoso, cruel y malo por la violencia; se identificaron respuestas enfocadas en la incertidumbre donde visualizan el futuro como incierto, no seguro, incierto en ámbitos escolares o laborales. Los estudiantes le atribuyen un valor muy importante al futuro, porque definirá su vida, donde se debe de reflexionar, tomar decisiones para poder identificar oportunidades y alcanzar metas. El futuro como oportunidad también aparece en el discurso de los estudiantes, colocando diferentes posibilidades para crear, lograr y vivir. El futuro lo ven como una consecuencia de sus actos, dependiendo de las opciones que se les presenten, la experiencia, aprendizaje, las elecciones y resultado de sus esfuerzos.

El futuro como consecuencia fue de las dimensiones que obtuvo un mayor número de respuestas, en ella los estudiantes manifestaron la responsabilidad que tienen en la construcción de su futuro, a través de sus actos presentes que repercutirán posteriormente.

El futuro se relaciona con el logro de una superación personal, basadas en construcción de metas. La perspectiva femenina muestra dimensiones parecidas a la de los hombres, sin embargo se agregó una nueva referente a los otros; es decir, futuro con base en otras personas, en este caso fueron los bebés de las estudiantes.

La dimensión del futuro como negativo sólo se observó en una respuesta relacionandolo con lo complicado. El aspecto positivo del futuro se concentró como una etapa de ventajas, relacionada con proyectos, estabilidad, prosperidad y ayuda, se visualizó como un tiempo bueno. La incertidumbre también fue evidente en el caso de las mujeres, el futuro como algo incierto, inseguro, inestable e impredecible. También apareció como un tiempo dado, una transición, algo próximo y como algo presente; también se le agregó un valor determinado, atribuyendo la caracteristica de algo muy importante que se debe de planear. Como oportunidad también fue evidente en el caso de las mujeres considerandolo como un espacio para alcanzar las metas. Como consecuncia apareció con gran peso, debido a que las estudiantes consideraron que son muy importantes sus acciones presentes como derivación en su futuro. Las acciones tuvieron mayores menciones en el caso de las mujeres, estuvieron principalmente enfocadas en actividades relacionadas con el estudio y cumplir metas. Los ideales de las mujeres se concentran en terminar los estudios, continuar estudiando, formar una familia, vida saludable, una buena posición económica, así como tener éxito en el ambiente laboral y personal. Se encontraron dos respuestas de estudiantes, una con un bebé, quien visualizó el futuro con lo mejor para su bebé; la otra estudiante se enfocó en su embarazo y su novio. 


\section{Valoración de la institución educativa}

En el discurso de los jóvenes es evidente la valoración que hacen los estudiantes sobre la institución educativa, en primer lugar aparece como ente que otorga o entrega conocimientos y los estudiantes los reciben o adquieren. La escuela les otorga aprendizaje y fortalece sus conocimientos, les ofrece herramientas para enfrentarse a las exigencias escolares y sociales.

Entre las ventajas de la escuela se encuentran las becas, el apoyo de profesores, los talleres, la vinculación con otras escuelas, los concursos, las prácticas y el servicio social. El capital escolar se relaciona con el ámbito laboral, abriendo un panorama de opciones con mejores sueldos y mayores ventajas en comparación con otros jóvenes. El bachillerato tecnológico les permite obtener un certificado de estudios y una cédula que los acredita como técnicos en las especialidades de Laboratorista Químico, Contabilidad, Programación y Mantenimiento Automotriz. El valor que se le otorga al certificado es instrumental, se ve como un pase de acceso al mundo laboral. Los estudiantes buscan terminar el bachillerato para poder adquirir el certificado y trabajar, se le atribuye al documento un valor muy importante, puesto que consideran que al adquirirlo será más sencillo obtener un empleo, lo cual generará independencia y la posibilidad de valerse por sí mismos.

En el siguiente ejemplo se evidencian las valoraciones respecto a la institución educativa:

\footnotetext{
"Ya tenemos una carrera técnica, ya podemos valernos de nosotros mismos para salir adelante, pues se supone que es para eso por lo que entramos al CETIS la verdad, porque ya salimos con una carrera técnica y ya si no queremos poder seguir estudiando pues con esa podemos, la mayoría eso es lo que piensa”. Mujer, Laboratorista Químico, 18 años.
}

Anteriormente se habló de que los estudiantes le atribuyen un valor muy importante al certificado, por la relación directa que consideran que existe entre el documento y mayor posibilidad de encontrar un trabajo; sin embargo, también es evidente que ellos se reconocen como estudiantes, los cuales deben tener mejores oportunidades en comparación con otros jóvenes que no se encuentran estudiando.

Los discursos evidencian una dimensión de "nosotros" los estudiantes y los "otros", personas que no tienen estudios, a quien frecuentemente hacen alusión los estudiantes y a los cuales desacreditan por no encontrarse en la misma posición, consideran que ellos deberían de tener mejor oportunidades que una persona que no estudió, porque ellos se están esforzando en sus estudios.

Los estudiantes se relacionan con palabras como superación, mejor calidad de vida, mejor trabajo, y a las personas que no estudian los describen como personas estancadas, que no quieren superarse y a las cuales las ven como modelos de futuro temido. 


\section{El estudiante como creador de su futuro}

Los estudiantes hicieron alusión a la responsabilidad que tienen en la construcción de sus expectativas de futuro y sobre todo en las acciones para cumplirlas, consideran que de ellos debe salir la inspiración para querer superarse, así como aprovechar las oportunidades para lograrlo; los estudiantes mencionaron a sus modelos a seguir o a personas que los inspiran, entre las que destacan la familia, amigos y personas normales que han sobresalido a pesar de su contexto y obstáculos, así como los artistas y personas exitosas, también consideran que las personas que tratan de frenarlos son una motivación para alcanzar las metas propuestas en los proyectos de vida.

A continuación mostramos un fragmento que ilustra las motivaciones:

"En mi caso yo misma, lo que me inspira y por decir, los grandes por decir, los que han inventado muchas cosas como las obras y eso, me inspiro en ellos, de que pues todos tenemos la capacidad de hacer algo, solamente hay que desarrollarlo". Mujer, Mantenimiento, 18 años.

En el discurso también fue evidente como los estudiantes se colocan como responsables de su futuro, atribuyendo a su ego una especie de motivador que los impulsa a cumplir sus metas, la responsabilidad de lograr sus objetivos sólo depende de ellos.

"En mi caso yo no tengo a nadie que me apoye, me apoyo yo mismo en lo que quiero". Hombre, Mantenimiento Automotriz, 18 años.

También se encontraron fragmentos donde se concentra el poder de decisiones en los mismos jóvenes a pesar del contexto.

"Cuando uno quiere algo o sea de verdad a pesar de los problemas, uno sobresale y sabe ya el camino para llegar a lo que realmente quiere". Mujer, Programación, 18 años".

\section{Futuro y las instituciones}

La escuela es uno de los elementos que influye en la configuración de las expectativas; sin embargo, podemos encontrar más elementos como los medios de comunicación, respecto a la pregunta por qué decidieron estudiar un bachillerato tecnológico un alumno contesto: "yo pensaba que iba a entrar al CETIS e iba a salir bien ching...". Hombre, Mantenimiento Automotriz, 17 años.

El epígrafe anterior es una frase que se rescató de uno de los informantes de la segunda entrevista colectiva, tenía puesta la valoración de la escuela en ideales que le generaron los medios de comunicación. El estudiante se encuentra en la especialidad de Mantenimiento Automotriz, la frase anterior hace referencia a que él consideró que su especialidad lo haría desarrollar las habilidades 
como en la película "rápido y furioso". Sin embargo, en palabras del estudiante, comentó que sufrió una desilusión porque aunque sí aprende en la escuela, necesita más práctica y no tanta teoría.

La institución educativa adquiere diferentes valoraciones para los estudiantes, en primer lugar se observó que le otorgan un valor a los conocimientos que adquieren a través de la escuela. Los discursos de los jóvenes manifestaron como ventaja la modalidad ambivalente del bachillerato tecnológico, ellos están conscientes que están realizando una carrera "corta", donde adquieren un certificado de una carrera técnica, que les podría asegurar un trabajo y mejores oportunidades.

Los estudiantes señalaron las metas que tenían al momento de entrar al bachillerato, en el caso de los hombres mencionaron objetivos enfocados en dimensiones académicas, donde su principal meta era terminar el bachillerato, con buen promedio, adquirir conocimientos de su especialidad para poder seguir estudiando, ingresar a una universidad. Otra dimensión que sobresale se relaciona con lo laboral, donde los estudiantes consideran como meta obtener un trabajo; en la dimensión social relacionada con la interacción con otras personas, se observan metas como: tener novia y amigos; en las metas personales buscan una superación, ser el mejor, buena persona y autosuficientes.

En el caso de las mujeres también tenían claro que quieren terminar el bachillerato, con un buen promedio, ingresar a la universidad y prepararse para la vida. En la dimensión laboral consideraron que una meta sería obtener un empleo; en lo social esperan tener novio y amigos, durante la estancia en el bachillerato; en la dimensión personal quieren superarse, formar una familia, sobresalir en la vida y tener una mejor condición de vida.

\section{Futuro ideal}

En el caso de los estudiantes que realizaron la entrevista colectiva, se les cuestionó sobre su futuro ideal. Cabe destacar que hicieron énfasis en tres ejes: laboral, personal y escolar.

El eje laboral, mostró un consenso respecto a las características que los estudiantes consideraron que debe de tener el trabajo ideal. Entre los adjetivos que más se repitieron se encontraron: buen trabajo, bueno y estable, buen sueldo, trabajo bien, trabajo estable y vida estable.

En el ámbito familiar siete jóvenes se ven con familia, sólo la mujer de Mantenimiento Automotriz, señaló que no se visualiza con familia propia.

En el ámbito escolar se encontraron discursos de los jóvenes con deseos de continuar sus estudios.

"Pues, yo me veo con un futuro pues ya, haciendo valer mi profesión, sería licenciatura, tener un buen sueldo. Hombre, Mantenimiento Automotriz, 18 años.

"Pues yo quiero continuar estudiando quiero terminar mi licenciatura en nutrición y de ahí empezar a ejercer lo que estudié y tener un trabajo estable”. Mujer, Laboratorista Químico, 17 años. 
El futuro ideal de los jóvenes reflejó estereotipos de personas, así como estilos de vidas comúnmente aceptables.

"Pues yo en la manera profesional pues concluir mis estudios, ya después trabajar un tiempo y ya después trabajar para mí mismo". Hombre, Programación, 17 años.

"Más que nada yo me veo como una persona de bien, donde yo trato de ser original, no una simple copia de una persona, donde profesionalmente me veo con una carrera ya terminada, laboralmente en un trabajo estable". Hombre, Laboratorista Químico, 17 años.

El discurso de los jóvenes muestra el deseo de continuar con sus estudios y sobre todo dedicarse a trabajar en lo que estudiaron. Los estudiantes coinciden en que un elemento para tener un futuro ideal es hacer lo que les guste:

"Yo creo que siempre debo de tratar de hacer lo que me guste y lo que yo en realidad quiero y no tratar de seguir a otras personas o así”. Mujer, Laboratorista Químico, 17 años.

\section{Futuro temido}

Los estudiantes también visualizaron un escenario de futuro temido, en el cual consideraron que no les gustaría estar. Entre las principales menciones aparecieron perderse en los vicios o adicciones. También resultó relevante que hicieron mención a seguir lo que las otras personas dicen o no hacer lo que a ellos les gusta.

\footnotetext{
"Pues así como no hacer lo que a mí me gusta, así como dejarme llevar por otras personas como lo que la sociedad dice que es lo ideal y dejarme llevar y no hacer lo que a mí me gusta y yo sé que es correcto". Mujer, Laboratorista Químico, 17 años.

"No sé algo negativo seria tratar de satisfacer las opiniones que los demás quieran antes que las mías, y si se diera el caso de que fuera así pues sería un futuro negativo, no me gustaría lo que hiciera aunque lo tuviera que hacer". Hombre, Contabilidad 17 años.
}

En el caso de las mujeres el futuro temido está relacionado con los frenos en su carrera profesional, al momento de convertirse en madre y que el esposo no la deje trabajar y que el tiempo que dedicó a estudiar no obtenga resultados enfocados en un buen trabajo. También consideran que el futuro temido sería estancarse o no superarse. 


\section{Conclusiones}

El principal hallazgo en el siguiente artículo fue que los jóvenes, en contextos educativos, sí piensan en su futuro, reflexionan y se cuestionan sobre diferentes dimensiones sociales, económicas, educativas, personales, en las cuales tienen que tomar decisiones y actuar. A pesar de que existen similitudes en las expectativas de hombres y mujeres, se observó que la variable de género modificó los resultados, después de obtener un buen trabajo, los hombres lo relacionan con el éxito y desean formar una familia, las expectativas de los hombres refuerzan su estatus, mostrando imágenes de futuro relacionadas con vidas deseables. En el caso de las mujeres se enfocan más en la autorrealización, enfocando ambientes económicos y personales.

La institucionalización de las expectativas de futuro se aprecia al analizar el discurso de los jóvenes, donde predominan adjetivos como bien, bueno, mejor, mostrando un discurso hegemónico relacionado con actitudes, comportamientos comúnmente aceptados como terminar la escuela, estudiar una licenciatura, obtener un buen promedio, adquirir un empleo, casarse y ser una buena persona, así como tener una vida bien. Caso opuesto el futuro temido está formado con no hacer lo que a los estudiantes les apasiona, con no desarrollar o aplicar las habilidades y competencias adquiridas en su carrera, con conductas, comportamientos y profesiones no aceptadas. Sin embargo, no toda la responsabilidad recae en la escuela para la formación de los estos ideales, los medios de comunicación y la familia juegan un papel muy importante debido a que la identidad de los estudiantes no es estática y se encuentra en constante cambio, la cual se nutre de diferentes redes sociales en las cuales se encuentra inserto.

La educación se sigue considerando como un recurso de movilidad social, los estudiantes creen que al adquirir el capital escolar, se tendrá un buen trabajo y se logrará el éxito. Los alumnos se consideran en doble ventaja en comparación con los que no estudian, porque los alumnos podrán insertarse al mundo laboral o continuar sus estudios, por lo que el valor de terminar el bachillerato es instrumental para adquirir el certificado y la cédula.

El discurso de los alumnos muestra una desacreditación hacia los "otros", es decir, hacia los jóvenes que no se encuentran en contextos escolares, los cuales son colocados en una posición de inferioridad, porque ellos no quieren superarse a través del estudio, los "otros" son vistos como jóvenes estancados, sin deseos o expectativas.

\section{Bibliografía}

Angenot, M. (2010). El discurso social. Los límites de lo pensable y lo decible. Buenos Aires: Siglo XXI.

Ávalos, J. (2007). La vida juvenil en el bachillerato. Una mirada etnográfica. México: Centro de Investigación y de Estudios Avanzados del Instituto Politécnico Nacional.

Bandura, A. (1999). Auto- Eficacia: cómo afrontamos los cambios de la sociedad actual. (J. Aldekoa, trad.). Bilbao: Desclée de Brouwer. 
Bourdieu, P. y J. Passeron (1981). La reproducción. Elementos para una teoría del sistema de enseñanza ( $2^{\mathrm{a}}$ ed.). París: Ediciones Minuit.

Dator, J. (2005). "Universities without 'quality'and quality without 'universities"'. On the Horizon. Vol. 13, núm. 4, pp. 199-215.

Davies, P. (1996). Sobre el tiempo: la revolución inacabada de Einstein. Barcelona: Editorial Crítica.

De la O, María Eugenia y Alma Leticia Flores Ávila (2012). "Violencia, jóvenes y vulnerabilidad en la frontera Noroeste de México". Desacatos, núm. 38. México: CIESAS.

María Eugenia de la O, Alma Leticia Flores Ávila (2012). "Violencia, jóvenes y vulnerabilidad en la frontera noreste de México". En: Desacatos, núm. 38, enero-abril. México: Centro de Investigaciones y Estudios Superiores en Antropología Social (CIESAS), pp. 11-28.

Dubet, François y Martuccelli, Danilo (1999): ¿En qué sociedad vivimos? Buenos Aires: Losada.

Garro-Rojas, L. (2006). Estigmas, miedos e imaginario de futuro: la construcción de identidades juveniles en un contexto de pobreza. San José, Costa Rica. Tesis doctoral, Doctorado en Estudios Científico Sociales. Tlaquepaque, Jalisco: ITESO.

Geertz, C. (1966). "Religion as a Cultural System". En: Anthropological Approaches to the Study of Religion. Conference on New Approaches in Social Anthropology. Nueva York: Praeger, pp. 1-46.

Guerra Ramírez, M. I. (2000). “QQué significa estudiar el bachillerato? La perspectiva de los jóvenes en diferentes contextos socioculturales". Revista Mexicana de Investigación Educativa. Vol. 5, núm. 10, julio-diciembre, pp. 243-272.

Toffler, A. (1973). El shock del futuro. Barcelona: Fondo de Cultura Económica.

Recibido: 02/10/13

Dictaminado: 23/01/14

Corregido: 04/03/14

Aceptado: 04/03/14 